\title{
APPROXIMATION OF NORMAL ELEMENTS IN THE MULTIPLIER ALGEBRA OF AN AF $C^{*}$-ALGEBRA
}

\author{
TERRY A. LORING AND JACK SPIELBERG
}

(Communicated by Palle E. T. Jorgensen)

\begin{abstract}
It is shown that there is a simple separable AF algebra $A$ such that $M(\mathscr{K} \otimes A)$ does not have weak (FN) and such that the generalized Berg-Weylvon Neumann Theorem does not hold for $\mathscr{K} \otimes A$.
\end{abstract}

Consider two properties enjoyed by every selfadjoint element $h$ of $L(H)=$ $M(\mathscr{K})$.

(1) $h$ can be approximated in norm by selfadjoint elements with finite spectrum.

(2) There is a sequence of mutually orthogonal compact projections $\left(e_{n}\right)$ and a bounded sequence of real numbers $\left(\lambda_{n}\right)$ such that $h-\sum_{n=1}^{\infty} \lambda_{n} e_{n} \in \mathscr{K}$ (the Weyl-von Neumann Theorem).

A $C^{*}$-algebra is said to be of real rank zero if (1) holds for every selfadjoint element $h$. The generalized Weyl-von Neumann Theorem for a $C^{*}$-algebra $A$ states that (2) holds for every selfadjoint element in the multiplier algebra $M(A)$, where $\mathscr{K}$ is replaced by $A$ (and the projections $\left(e_{n}\right)$ belong to $A$ and sum to the identity). These generalizations of (1) and (2) have attracted much attention in recent years. In particular, when $A$ is $\sigma$-unital and has real rank zero, it has been shown that $M(A)$ has real rank zero if and only if the generalized Weyl-von Neumann Theorem holds for $A$ [Lin1, Zha]. Moreover, Lin has shown that if $A$ is a $\sigma$-unital AF algebra, then $M(A)$ has real rank zero [Lin2]. As AF algebras are in many ways the simplest generalization of the algebra $\mathscr{K}$, this result is very encouraging.

Consider now analogues of properties (1) and (2) for normal operators. A $C^{*}$-algebra is said to have property $(F N)$ if every normal element can be approximated in norm by normal elements having finite spectrum. By the spectral theorem, $L(H)$ has $(\mathrm{FN})$. In more general multiplier algebras this definition is too strong. In a $C^{*}$-algebra whose $K_{1}$ group is nontrivial, an element $x$ might fail to be approximated by elements with finite spectrum due to index obstructions corresponding to holes in the spectrum of $x$. Lin has defined weak $(F N)$ to take such obstructions into account [Lin3, Definition 4.2]. Without recalling

Received by the editors November 9, 1992.

1991 Mathematics Subject Classification. Primary 46L05.

The first author was partially supported by National Science Foundation grant DMS-9007347. The second author was partially supported by National Science Foundation grant DMS-9102971. 
the definition, we remark that if an algebra has weak (FN) then a normal element with spectrum equal to a disk can be approximated by normal elements with finite spectrum.

Berg's generalization of the Weyl-von Neumann Theorem states that (2) holds for every normal operator $h$ in $L(H)$, where the $\left(\lambda_{n}\right)$ are allowed to be complex [Ber]. There are several generalizations of this to multiplier algebras. We will use the following

Definition. Let $A$ be a $C^{*}$-algebra. We say that the generalized Berg-Weyl-von Neumann Theorem holds for $A$ if the normal elements of $M(A)$ are quasidiagonal (cf. [Zha, 1.3]); i.e., given any normal element $h$ in $M(A)$ there is a sequence of mutually orthogonal projections $\left(e_{n}\right)$ in $A$ and a bounded sequence $\left(a_{n}\right)$ in $A$ such that

(i) $\sum e_{n}=1$,

(ii) $a_{n}=e_{n} a_{n} e_{n}$ for all $n$,

(iii) $h-\sum a_{n} \in A$,

where the sums in (i) and (iii) are taken in the strict topology.

The result of this note is to point out the following

Theorem 1. There is a simple separable $A F$ algebra $A$ such that $M(\mathscr{K} \otimes A)$ does not have weak $(F N)$ and such that the generalized Berg-Weyl-von Neumann Theorem does not hold for $\mathscr{K} \otimes A$.

We first prove the more general

Theorem 2. Let $A$ be a $C^{*}$-algebra admitting a ${ }^{*}$-homomorphism $\varphi: C_{0}\left(\mathbf{R}^{2}\right) \rightarrow$ $A$ such that $\varphi_{*}: K_{0}\left(C_{0}\left(\mathbf{R}^{2}\right)\right) \rightarrow K_{0}(A)$ is nonzero. Then $M(\mathscr{K} \otimes A)$ does not have weak $(F N)$ and the generalized Berg-Weyl-von Neumann Theorem does not hold for $\mathscr{K} \otimes A$.

Proof. By Theorems 1 and 2 of [MS] there is a normal element $h \in$ $M(\mathscr{K} \otimes A)$ such that the spectrum of $h$ is the closed unit disk, $\pi(h)$ is unitary in $M(\mathscr{K} \otimes A) / \mathscr{K} \otimes A$, and $\partial[\pi(h)]=\varphi_{*}(b)$, where $\pi$ is the quotient map, $\partial$ is the connecting map in $K$-theory, and $b$ is the generator of $K_{0}\left(C_{0}\left(\mathbf{R}^{2}\right)\right)$. It follows that $\pi(h)$ cannot be norm-approximated by invertibles with finite spectrum and, hence, that $h$ cannot be norm-approximated by elements with finite spectrum. As remarked earlier, this implies that $M(\mathscr{K} \otimes A)$ does not have weak (FN).

Now suppose that the generalized Berg-Weyl-von Neumann Theorem holds for $\mathscr{K} \otimes A$. Let $\left(e_{n}\right)$ and $\left(a_{n}\right)$ be as in the above definition for the element $h$. Let $x=h-\sum a_{n}$. Since $\pi(h)$ is unitary, we have

$$
\sum\left(e_{n}-a_{n}^{*} a_{n}\right)=1-(h-x)^{*}(h-x) \in \mathscr{K} \otimes A
$$

and, similarly, $\sum\left(e_{n}-a_{n} a_{n}^{*}\right) \in \mathscr{K} \otimes A$. Therefore, $\left\|e_{n}-a_{n}^{*} a_{n}\right\| \rightarrow 0$ and $\left\|e_{n}-a_{n} a_{n}^{*}\right\| \rightarrow 0$. It follows that, for large enough $n, y_{n}=\sum_{k=1}^{n} e_{k}+\sum_{k=n+1}^{\infty} a_{k}$ is invertible. Since $h-y_{n} \in \mathscr{K} \otimes A$, we obtain the contradiction

$$
\partial[\pi(h)]=\partial\left[\pi\left(y_{n}\right)\right]=\partial \circ \pi_{*}\left[y_{n}\right]=0 .
$$

Proof of Theorem 1. Elliott and Loring have shown that a simple unital AF algebra admits a ${ }^{*}$-homomorphism $\varphi$ as in the statement of Theorem 2 if and only if its dimension group contains nonzero elements in the intersection of the 
kernels of all the finite traces [EL]. Simple dimension groups containing such elements abound and provide examples verifying Theorem 1 .

Specifically, they have a quite simple explicit example, which appears in $\S 6$ of [Lor 1]. The stationary inductive system given by the matrix $\left(\begin{array}{ll}2 & 1 \\ 1 & 2\end{array}\right)$ defines a simple AF algebra whose dimension group is $\left\{\left(m / 3^{n}, k\right) \mid m \equiv k(\bmod 2)\right\} \subseteq$ $\mathbf{Z}\left[\frac{1}{3}\right] \oplus \mathbf{Z}$, with strict order from the first coordinate.

Remark. For an example of a separable $C^{*}$-algebra having real rank zero but not having weak (FN), see [Lor2].

\section{REFERENCES}

[Ber] I. D. Berg, An extension of the Weyl-von Neumann Theorem to normal operators, Trans. Amer. Math. Soc. 160 (1971), 365-371.

[EL] G. A. Elliott and T. A. Loring, $A F$ embeddings of $C\left(T^{2}\right)$ with prescribed $K$-theory, J. Funct. Anal. 103 (1992), 1-25.

[Lin1] H. Lin, On ideals of multiplier algebras of simple $A F C^{*}$-algebras, Proc. Amer. Math. Soc. 104 (1988), 239-244.

[Lin2] _ Generalized Weyl-von Neumann theorems, Internat. J. Math. 2 (1991), 725-739.

[Lin3] _ Approximation by normal elements with finite spectra in $C^{*}$-algebras of real rank zero, preprint.

[Lor1] T. A. Loring, Berg's technique for pseudo-actions with applications to AF embeddings, Canad. J. Math. 43 (1991), 119-157.

[Lor2] _-, Normal elements of $C^{*}$-algebras of real rank zero without finite-spectrum approximants, J. London Math. Soc. (to appear).

[MS] J. A Mingo and J. S. Spielberg, The index of normal Fredholm elements of $C^{*}$-algebras, Proc. Amer. Math. Soc. 113 (1991), 187-192.

[Zha] S. Zhang, $K_{1}$-groups, quasidiagonality, and interpolation by multiplier projections, Trans. Amer. Math. Soc. 325 (1991), 793-818.

Department of Mathematics and Statistics, University of New Mexico, Albuquerque, New MeXico 87131

E-mail address: loring@math.unm.edu

Department of Mathematics, Arizona State University, Tempe, Arizona 85287

E-mail address: jss@math.la.asu.edu 